\title{
Vers une caractérisation des exercices de français pour italophones. Le cas des articles partitifs
}

Towards a characterization of French exercises for Italian speakers. The case of partitive articles

Raphaële Fouillet

\section{OpenEdition}

\section{Journals}

Édition électronique

URL : https://journals.openedition.org/dhfles/6986

DOI : $10.4000 /$ dhfles.6986

ISSN : 2221-4038

Éditeur

Société Internationale pour l'Histoire du Français Langue Étrangère ou Seconde

Édition imprimée

Date de publication : 1 décembre 2019

Pagination : 497-518

ISBN : 0992-7654

ISSN : 0992-7654

Référence électronique

Raphaële Fouillet, «Vers une caractérisation des exercices de français pour italophones. Le cas des articles partitifs », Documents pour l'histoire du français langue étrangère ou seconde [En ligne], 62-63 | 2019, mis en ligne le 12 avril 2020, consulté le 27 mars 2023. URL : http://journals.openedition.org/ dhfles/6986 ; DOI : https://doi.org/10.4000/dhfles.6986

Ce document a été généré automatiquement le 27 mars 2023

Tous droits réservés 


\title{
Vers une caractérisation des exercices de français pour italophones. Le cas des articles partitifs
}

\author{
Towards a characterization of French exercises for Italian speakers. The case of \\ partitive articles
}

Raphaële Fouillet

\section{Introduction}

Les plus anciens manuels de français édités dans la péninsule italienne remontent au XVII $^{\mathrm{e}}$ siècle. D'après Nadia Minerva et Carla Pellandra ${ }^{1}$ (1991: 21), l'apparition et la systématisation des exercices dans les livres de français pour italophones se situent dans la première moitié du XIX ${ }^{\mathrm{e}}$ siècle, vers 1820 plus exactement. L'objet de cet article est de proposer une caractérisation provisoire des exercices conçus pour des locuteurs de l'italien, au moyen de l'analyse à titre exploratoire d'un corpus portant sur la classe grammaticale des articles partitifs. Dans la perspective d'obtenir un aperçu de l'évolution des types d'exercice proposés par différents auteurs, une série d'ouvrages a été sélectionnée de manière aléatoire pour constituer un corpus jalonnant les deux siècles nous séparant des premiers exercices créés pour accompagner les descriptions grammaticales. Après une présentation des manuels et des recueils dont est tiré le corpus, nous examinerons les types d'exercice le constituant sur la base de la typologie de Gérard Vigner (2016), pour ensuite analyser sous l'angle pédagogique les exercices proposés autour de l'article partitif. 


\section{Une brève présentation du corpus}

2 Le corpus est constitué d'exercices extraits de trois manuels de langue française et de quatre recueils d'exercices. Les ouvrages ont été choisis au hasard de leur accessibilité, avec malgré tout l'objectif de balayer presque deux siècles d'édition de manuels de français comportant des exercices.

3 Le premier ouvrage dans l'ordre chronologique est de Salvatore Torretti. Son Corso completo di lingua francese ad uso degl'Italiani ovvero grammatica francese datant de 1823 a connu au moins onze publications entre 1823 et 1858 . Rédigé en italien, il égrène les classes grammaticales sous forme de parties possédant chacune un titre. Chaque règle ${ }^{2}$ morphosyntaxique est numérotée; de nombreux exemples illustrent les descriptions grammaticales. Ceux-ci sont présentés en colonne et en miroir : à gauche les exemples en italien et à droite, leur équivalent en français. Les exercices proposés se situent à la fin de chaque point de grammaire traité.

4 Le deuxième ouvrage consulté, Nuova grammatica teorico-pratica della lingua francese, est publié pour la première fois par Ferdinando Bassi en 1896 et connaîtra plusieurs éditions au cours de la première moitié du $\mathrm{XX}^{\mathrm{e}}$ siècle. Écrit en italien, celui-ci est divisé en leçons à la suite desquelles se trouvent les exercices.

5 Le recueil d'exercices de Carlo Truchi (1949) est le troisième ouvrage dont est tiré le corpus. Rédigé en italien et en français, Raccolta di esercizi e temi graduati di lingua francese ad uso delle scuole medie se compose de trois parties: la première est consacrée aux esercizi di traduzione sulle regole di morfologia, la seconde aux exercices sur les règles de syntaxe et la troisième aux temi di ricapitolazione generale ${ }^{3}$.

Le volume Esercizi di francese de Luciano Bosisio paru en 1949 est notre quatrième ouvrage. Exclusivement écrit en italien, il accompagne un volume de grammaire. Quant à l'ouvrage de Giacomo d'Alessandro imprimé en 1973, le cinquième dont est extrait le corpus, il est constitué de plusieurs parties: l'une est dédiée aux esercizi sulla grammatica, une deuxième aux temi progressivi sulla grammatica, une troisième aux esercizi sulla sintassi et une quatrième aux temi di traduzione. Passi di autori italiani. Les consignes des exercices sont rédigées en italien ou en français.

7 La Grammaire du français pour italophones et les Exercices de grammaire, notre sixième ouvrage, publiés en 1994 par Françoise Bidaud font l'objet de deux volumes séparés. Ils sont entièrement écrits en français. Enfin, Grammaire en situation, notre dernier ouvrage, publié par Lisa Beneventi en 2011, est rédigé en italien. Divisé en actes de langage et suivant une progression conforme au CECRL, le manuel propose des exercices à la fin de chaque double page consacrée à un point de grammaire.

\section{Quels exercices dans les manuels de français pour italophones?}

8 D'après Henri Besse et Rémy Porquier (1991: 120), l'exercice en classe de langue présente quelques traits distinctifs : c'est " une tâche ${ }^{4}$ langagière précise " s'inscrivant dans le «rituel communicatif propre à la classe»; c'est une tâche répétitive et contrainte par un cadre donné par une consigne; c'est une activité métalinguistique centrée sur un aspect partiel de la langue. Au-delà de ces caractéristiques générales, G. 
Vigner (2016) a élaboré une typologie qui sert de point de départ à notre proposition de caractérisation des exercices de français pour italophones.

\section{La typologie de Gérard Vigner (2016)}

9 G. Vigner retient six types d'exercice :

- les exercices lacunaires ou exercices à trous où l'apprenant complète des phrases au moyen d'éléments linguistiques donnés en liste fermée ;

- les exercices à choix multiples dans lesquels l'apprenant doit choisir une réponse parmi plusieurs possibilités ;

- les exercices à dominante morphologique où l'apprenant doit conjuguer ou accorder ;

- les exercices de transformation dans lesquels l'apprenant modifie des phrases en passant d'une forme à une autre ;

- les exercices de mise en ordre qui consistent à remettre dans l'ordre des mots afin de former une phrase ;

- les exercices de reconnaissance grammaticale où il est demandé à l'apprenant de reconnaître une forme grammaticale et de justifier sa réponse.

On remarquera que cette typologie ne permettra pas toujours de classer tous les exercices rencontrés dans les manuels pour italophones.

\section{Occurrences et usages du mot « exercice »}

11 Les auteurs des ouvrages consultés emploient le mot "exercice " pour désigner différentes tâches proposées à l'élève. Dans la page de titre de l'ouvrage de $\mathrm{S}$. Torretti (1823), le terme « exercice » est attaché à la seule prononciation. L'autre type de tâche proposé à l'élève est celle du " thème de français ». Près d'un siècle plus tard, le titre de la grammaire de F. Bassi annonce en 1912 des «exercices de traduction, de conversation, de lecture et de composition ». Il s'agit de quatre types de tâche qu'il nomme ainsi :

- la versione orale o scritta dont l'appellation induit la tâche à effectuer ;

- l'esercizio scritto pour lequel il n'est donné aucune consigne et qui présente une succession de phrases en italien qu'il s'agit vraisemblablement de traduire ;

- la ripetizione qui consiste en questions grammaticales en italien sur la leçon;

- la conversation grammaticale, qui se présente sur deux colonnes : dans la colonne de gauche, les principales questions posées en italien dans la tâche précédente sont reformulées en français et dans la colonne de droite, sont fournies les réponses.

L. Bosisio publie en 1949 un ouvrage nommé Esercizi di lingua francese où le terme "exercice » désigne un ensemble de phrases à traduire de l'italien vers le français à l'écrit et à l'oral. C. Truchi, quant à lui, fait également paraître en 1949 un ouvrage entièrement consacré à la pratique dont le titre distingue d'un côté les « exercices » et de l'autre les "thèmes ». Les exercices relèvent de la morphologie d'une part et de la syntaxe d'autre part: les premiers consistent en une succession de phrases décontextualisées à traduire et les seconds regroupent des exercices lacunaires, des exercices de traduction et des exercices à dominante morphologique.

G. d'Alessandro publie également en 1973 un ouvrage entièrement consacré à la pratique dont le sous-titre est très proche de celui de $C$. Truchi. Il distingue de la même manière les exercices et les thèmes. Des exercices sont consacrés « à la grammaire » et 
d'autres « à la syntaxe». Pour chaque exercice, il s'agit de traduire une succession de phrases sans lien les unes avec les autres, hormis le point de grammaire travaillé.

On relève deux caractéristiques communes aux ouvrages cités jusqu'à présent: l'absence de consigne et la distinction de chaque exercice par un titre désignant un fait de langue comme "forme passive et complément d'agent» ou encore "accord de l'adjectif avec le nom ».

15 Les deux ouvrages les plus récents se distinguent radicalement des précédents dans le sens où les exercices ne sont plus annoncés au moyen d'un titre ou d'un nom mais au moyen de consignes qui permettent de les catégoriser en fonction des tâches à réaliser.

Nous avons ainsi relevé dans les Exercices de grammaire de F. Bidaud (1994), toujours d'après la typologie de G. Vigner (2016) mais à laquelle quelques types ont été ajoutés, les types suivants :

- des exercices à dominante morphologique, exemple de consigne : « Mettre les adjectifs entre parenthèse au féminin » $(1994: 12)$;

- des exercices lacunaires, exemple de consigne : «Compléter par les démonstratifs ce, cet ou cette » $(1994: 30)$;

- des exercices de transformation, exemple de consigne : «Transformer selon le modèle » (1994: 30);

- des exercices de production, exemple de consigne : « Présenter les personne suivantes selon les renseignements qui sont donnés. Préciser également l'année de naissance » (1994:56);

- des exercices de reconnaissance grammaticale, exemple de consigne: "Classer les différentes phrases de l'exercice précédent dans les trois catégories suivantes en indiquant les numéros des phrases » (1994:44);

- des exercices d'association, exemple de consigne: «Relier chaque verbe à un adjectif » (1994: 87);

- des exercices de traduction du français vers l'italien et de l'italien vers le français, exemple de consigne : « Traduire » $(1994: 56)$.

Chez L. Beneventi (2011), les tâches regroupées sous le terme «exercice " prennent globalement une dimension supplémentaire, celle de l'ancrage de la tâche à accomplir dans une situation de communication la plus réelle possible. L'ouvrage propose :

- des exercices de transformation, exemple de consigne : Rispondi negativamente alle domande $(2011: 69)$;

- des exercices lacunaires, exemple de consigne : Completa queste descrizioni con c'est $o$ il est $(2011: 39)$;

- des exercices de traduction de l'italien vers le français, exemple de consigne : Traduci questi enunciati in francese $(2011: 39)$;

- des exercices à dominante morphologique, exemple de consigne : Quali sono le abitudine di Medhi ? Completa il suo blog coniugando i verbi tra parentesi (2011:45);

- des exercices de production, exemple de consigne : Completa liberamente (2011:57) ;

- des exercices d'association, exemple de consigne : Associa domande e risposte (2011 : 73).

18 Le recensement des types d'exercice met en évidence une évolution en termes de diversification des tâches à effectuer mais aussi une constante : la traduction. 


\section{Exercice et traduction}

19 Suivant les auteurs, la traduction est classée sous la dénomination «exercice » ou «thème » et ne recouvre pas les mêmes objets. Pour F. Bidaud (1994) et L. Beneventi (2011), tout comme pour N. Minerva et C. Pellandra (1991a : 21), la traduction est un exercice comme un autre. La matière linguistique à traduire est constituée de phrases isolées chez les deux auteures, parfois tirées de textes littéraires. F. Bassi (1912) range également la traduction sous l'étiquette "exercice». Il propose des versions à faire à l'oral ou à l'écrit et des thèmes écrits.

Quant à S. Torretti (1823), on l'a vu plus haut, il distingue l'exercice où il s'agit de traduire des groupes de mots et le thème qui consiste à traduire des textes non littéraires en italien. Le point commun est que l'exercice aussi bien que le thème ont pour objectif de travailler un point de grammaire précis. L. Bosisio (1949) propose des phrases et des textes à traduire qu'il appelle "exercice» et des thèmes dits de "récapitulation" présents à la fin d'une série d'« exercices" de traduction. Il s'agit alors d'extraits de textes littéraires à traduire. Chez C. Truchi (1949) et G. d'Alessandro (1973), on trouve respectivement les temi di ricapitolazione generale et les temi progressivi sulla grammatica et les temi progressivi sulla sintassi ${ }^{5}$. Une dernière partie est cependant réservée à la traduction d'extraits d'œuvres littéraires dans le recueil d'exercices de G. d'Alessandro. On constate bien l'attachement à la traduction toujours présente dans le manuel le plus récent tout en observant un changement dans le type de texte à traduire : on passe de manière générale des extraits de textes littéraires ou de leçons de morale à des phrases plus ou moins contextualisées chez nos auteures contemporaines.

Outre la résistance de la traduction au passage du temps et aux grands changements didactiques dans l'enseignement des langues, on note un autre trait invariable dans le corpus d'exercices : l'approche contrastive.

\section{Le cas des articles partitifs}

Les articles partitifs constituent un point de contraste systématiquement décrit dans les manuels consultés. De S. Torretti (1823) à L. Beneventi (2011), il est mentionné. C'est la raison pour laquelle nous l'avons choisi pour analyser les exercices proposés en lien avec celui-ci.

\section{L'article partitif : descriptions}

Dès les premiers manuels de français à l'usage des Italiens, la description linguistique est en partie bâtie sur une comparaison entre les deux langues ${ }^{6}$. Nous qualifions de contrastive cette approche descriptive, même si elle ne relève pas strictement de l'analyse contrastive au sens strict de Robert Lado $^{7}$ (1971). Les différences et les ressemblances entre l'italien et le français mentionnées dans les manuels de français conçus pour les italophones sont certes structurelles, mais elles sont surtout fondées sur l'expérience des enseignants, auteurs des manuels, qui constatent qu'elles constituent un point d'achoppement pour les apprenants. de grammaire consultés de 1823 à 2011. Elles attirent l'attention de l'élève sur une 
différence structurelle entre les deux langues : là où l'italien supprime l'article partitif, le français en impose l'usage devant le nom pris au sens partitif. La formulation du contraste entre les deux langues varie suivant les auteurs mais la substance est la même :

« En italien on omet très souvent cet article, lequel est presque toujours exprimé en français ${ }^{8} »$ (Torretti $1823: 48$ ).

«En français, devant les noms pris dans un sens partitif, c'est-à-dire indiquant une partie ou une quantité indéfinie d'un tout, on emploie les particules du, de la, de l', des, c'est-à-dire la préposition de et l'article, même si en italien les particules correspondantes [les articles partitifs] sont sous-entendues, comme cela arrive très souvent» (Bassi 1912: 7).

«Rappelez-vous qu'en français les noms partitifs veulent toujours être précédés de l'article » (Truchi 1949 : 15).

"Le substantif partitif en italien peut être accompagné ou non de la préposition articulée ; en français normalement, il l'est » (Bosisio 1949a : 52).

«L'article partitif pose en outre des problèmes aux italophones parce qu'il est beaucoup moins utilisé en italien, qui, comme l'ancien français, préfère souvent l'article zéro » (Bidaud 1994 a : 33).

«L'article partitif peut aussi correspondre en italien à l'article défini ou à l'indéfini certo $"$ (Bidaud 1994a : 34).

«En italien le partitif est très souvent omis. En français, il doit toujours être exprimé » (Beneventi $2011: 84$ ).

Ces descriptions sont accompagnées dans tous les cas d'exercices exploitant ce point de contraste.

\section{L'article partitif : exercices}

Nous qualifions de contrastifs les exercices conçus pour répondre à une difficulté identifiée comme due à la divergence entre les deux langues. Les auteurs mobilisent des types d'exercice différents mis au service d'un objectif commun : travailler le point de contraste mis en évidence dans les descriptions grammaticales.

\section{Des exercices de traduction}

L'exercice de la traduction est le plus courant, de l'ouvrage de S. Torretti (1823) jusqu'aux Exercices de grammaire de F. Bidaud (1994). S. Torretti privilégie l'exercice du thème. Il en propose deux ${ }^{9}$ (Document 1 en annexe) sur l'article partitif. Il s'agit de textes en italien où tous les noms pris dans un sens partitif ne sont précédés d'aucun article. Deux actions sont demandées à l'élève : la première est de retrouver les noms devant lesquels il est possible d'ajouter en italien del, dello, della, dei, degli, delle et la seconde est d'insérer dans leur traduction la forme correspondante en français. L'auteur s'appuie donc ici sur la connaissance grammaticale de la langue italienne par l'élève et l'objectif semble être l'automatisation de l'emploi du partitif en français. C. Truchi (1949) utilise un type d'exercice identique mais en compliquant un peu plus la tâche de l'élève. Ce dernier doit en effet traduire en français plusieurs textes en italien dans lesquels se mêlent articles partitifs et preposizioni articolate. Il s'agit dans un premier temps de bien distinguer le partitif du complément de nom en italien pour ensuite traduire correctement les articles partitifs.

L'ouvrage de G. d'Alessandro (1979) contient également un exercice de traduction de l'italien vers le français intitulé Casi particolari del partitivo (Document 2 en annexe) où il 
mélange les cas : des syntagmes nominaux réclamant l'article partitif en français et des expressions requérant l'article zéro. Cet exercice affiche un degré de difficulté supplémentaire aux exercices de C. Truchi. De la même manière, F. Bidaud (1994) a conçu deux exercices de traduction de l'italien vers le français (Document 3 en annexe) où elle confronte les étudiants à plusieurs différences entre l'italien et le français : l'emploi de l'article partitif en français et de l'article zéro en italien mais aussi l'emploi de l'article partitif ou indéfini en français et de l'article défini en italien.

F. Bassi (1912) adopte une démarche différente. Il propose dans un premier temps une version orale ou écrite (Document 4 en annexe) dans laquelle les articles partitifs français sont mis en évidence par le choix de l'italique. L'élève est par conséquent guidé dans le repérage et l'emploi de l'article partitif en français. Devant traduire du français vers l'italien, il est censé saisir la différence d'emploi du partitif entre les deux langues mais également distinguer les articles partitifs des articles contractés en français grâce aux compléments de nom concomitamment présents dans le texte à traduire.

F. Bassi propose ensuite un " exercice écrit » (Document 5 en annexe) où il est question pour l'élève de traduire de l'italien vers le français des noms pris dans un sens partitif et qui ne sont précédés d'aucun article. L'objectif de cet exercice semble clairement de concentrer l'attention de l'élève sur la différence structurelle entre les deux langues, l'effort cognitif étant réduit au minimum par l'apport de la traduction des autres mots ou groupe de mots en bas de page. L. Bosisio (1949) opte également pour ce type d'exercice. Il en présente trois sur l'article partitif où il est demandé à l'élève de traduire des phrases italiennes en français (Document 6 en annexe).

Les objectifs de l'exercice de traduction sont multiples : ils vont de l'automatisation de l'utilisation du partitif à la mobilisation de connaissances fines sur les différents emplois des articles en français et en italien. Des degrés de difficultés en découlent: l'attention de l'élève peut être concentrée sur la simple forme des articles ou l'élève est amené à manipuler plusieurs fonctions et emplois des articles. Enfin, les auteurs s'appuient tous sur la connaissance de la grammaire italienne par les élèves. L'exercice de la traduction permet de " goûter » la différence structurelle concernant l'emploi de l'article partitif.

\section{Des exercices de mémorisation}

Bassi (1912) use d'un autre type d'exercice dont l'objectif principal est un apprentissage par cœur déguisé par la répétition à voix haute des règles de grammaire. Il s'agit de deux exercices où l'élève et le maître "dialoguent ", en italien puis en français, sur le thème de la leçon. Les questions 10 de la ripetizione et de la "conversation grammaticale » (Document 7 en annexe) ont pour objet le point de contraste entre les deux langues : "Que mettent les Français devant un nom pris dans un sens partitif ? ».

\section{Des exercices lacunaires}

33 Le type de l'exercice lacunaire apparaît chez F. Bidaud (1994) et surtout chez L. Beneventi (2011). Toutes les deux proposent des exercices où il s'agit de compléter des phrases en français par les articles partitifs adéquats. Dans l'exercice 57 (Document 8 en annexe), il s'agit essentiellement de faire travailler la forme de l'article partitif français. Dans l'exemple tiré de L. Beneventi (Document 9 en annexe), l'exercice lacunaire est un peu plus complexe dans la mesure où plusieurs cas sont présents : 
l'article partitif présent en français mais absent en italien; les quantitatifs " beaucoup ", " peu ", " trop » suivis de la préposition " de »; certaines prépositions se construisant avec l'article zéro, « des » qui devient « de » dans une phrase négative.

\section{Un exercice lacunaire original}

C. Truchi (1949) propose un type d'exercice hybride entre l'exercice lacunaire et la traduction (Document 10 en annexe) où il allie le fond et la forme dans la prise en compte d'un point de contraste entre l'italien et le français. L'exercice court sur plusieurs pages. Il se présente sous la forme d'un texte en italien où, sous chaque phrase, la traduction en français est écrite mais dans laquelle il faut ajouter l'article partitif qui est absent en italien. La mise en page de l'exercice souligne le souhait de l'auteur d'être au plus proche de la différence structurelle entre le français et l'italien. Tout comme chez Bassi, il oriente l'attention de l'élève sur le point de contraste en lui facilitant cognitivement la traduction du texte. Cet exercice qui semble être un banal exercice de traduction illustre à mon sens une forme d'inventivité de l'auteur qui a souhaité contextualiser la difficulté. La différence structurelle est en quelque sorte fondue dans un ensemble de phrases et devrait conduire l'élève à mieux saisir l'utilisation en contexte du partitif en français. La mise en miroir des deux langues devrait amener l'élève à saisir la différence structurelle et à automatiser par l'exercice de traduction l'usage du partitif en français.

\section{Des exercices de reconnaissance grammaticale}

Un dernier type d'exercice exploitant le point de contraste dans le cas de l'article partitif est présent chez F. Bidaud (Document 11 en annexe). Il s'agit d'un exercice à la fois lacunaire et de reconnaissance grammaticale où l'étudiant doit compléter des phrases en français à l'aide d'un article partitif ou d'un article contracté et identifier la nature des articles qu'il choisit. C'est un exercice qui fait appel à la fois à la réflexion grammaticale et à la connaissance métalinguistique de l'étudiant.

\section{Conclusion}

L'exercice qui se généralise dans les manuels de langue française à visée pédagogique pour les Italiens dans la première moitié du XIX siècle présente des caractéristiques à la fois constantes et évolutives. La transformation la plus manifeste réside dans la diversification des types d'exercice proposés : chez S. Torretti (1823), on ne trouve que des thèmes qui ne sont d'ailleurs pas catégorisés comme exercice. À partir de l'ouvrage de Bassi (1912), on remarque une tentative de varier les exercices, même si les leviers d'apprentissage mobilisés sont toujours la mémorisation, la répétition et la traduction. Cependant l'oral s'impose et occupe une place aussi importante que l'écrit dans la traduction et la répétition des exercices. Une véritable diversification s'opère dans les années 1990 et nombre d'exercices du corpus se retrouve dans la typologie de G. Vigner (2016). Avec le CECRL et l'ouvrage de L. Beneventi (2011), ils acquièrent une nouvelle dimension qui est celle de simuler des situations de communication réelles.

Cette diversification des types d'exercice s'accompagne de l'apparition de consignes, inexistantes dans les ouvrages consultés jusqu'à 1973 où il s'agit majoritairement de traduire. La traduction, absente de la typologie de G. Vigner (2016), est une constante 
du corpus. Elle est conservée jusqu'à aujourd'hui comme activité d'entraînement à la langue française et rapidement rangée dans la catégorie "exercice " par les auteurs italophones. On passe ainsi d'une tâche quasi unique qui est le thème au XIX ${ }^{\mathrm{e}}$ siècle à une diversification des exercices où se maintient malgré tout la traduction.

On relève par ailleurs une autre constante caractéristique des exercices de français pour italophones: l'approche contrastive. Cette dernière déjà présente dans les descriptions grammaticales l'est également dans les exercices. Le cas de l'article partitif illustre bien cette dimension: la différence structurelle entre le français et l'italien formulée dans les descriptions fait l'objet d'exercices ad hoc. L'approche contrastive a su épouser l'évolution et la diversification des types d'exercice et toujours occuper une place importante dans l'enseignement/apprentissage du français en Italie.

\section{BIBLIOGRAPHIE}

\section{Sources primaires}

\section{Manuels de français publiés en Italie}

ALESSANDRO (D’), Giacomo (1973). Je traduis - Je rédige. Vérone : Edizioni scolastiche Mondadori.

BASSI, Ferdinando (1912). Nuova grammatica teorico-pratica della lingua francese : con numerosi esercizi di traduzione, di conversazione, di lettura e di composizione accompagnati da una raccolta di proverbi e di gallicismi : ad uso delle scuole secondarie d'Italia. Trévise : Luigi Zoppelli.

BENEVENTI, Lisa (2011). Grammaire en situation. Bologne : Zanichelli.

BIDAUD, Françoise (1994a). Grammaire du français pour italophones. Florence : Scandicci.

BIDAUD, Françoise (1994b). Exercices de grammaire. Florence : Scandicci.

BOSISIO, Luciano (1949a). La grammatica francese del XX secolo. Vérone : Edizioni scolastiche

Mondadori.

Bosisio, Luciano (1949b). Esercizi di lingua francese. Vérone : Edizioni scolastiche Mondadori.

TORRETTI, Salvatore (1823). Corso completo di lingua francese ad uso degli Italiani ovvero grammatica francese. Milan : Giovanni Silvestri.

TORRETTI, Salvatore (1829). Corso completo di lingua francese ad uso degli Italiani ovvero grammatica francese. Milan : Giovanni Silvestri.

TRUCHI, Carlo (1949). Raccolta di esercizi e temi graduati di lingua francese ad uso delle scuole medie, Turin : Società Editrice Internazionale.

\section{Sources secondaires}

BESSE, Henri et PORQUIER, Rémy (1991). Grammaires et didactique des langues. Paris : Didier/Crédif. 
CUQ, Jean-Pierre (dir.) (2003). Dictionnaire de didactique du français langue étrangère et seconde. Paris : Asdifle/CLE International.

LADO, Robert (1971 [1957]). Linguistics across cultures - Applied Linguistics for Language Teachers. The University of Michigan Press.

MANDICH, Anna (2002). Insegnare il francese in Italia. Repertorio di manuali pubblicati in epoca fascista (1923-1943). Bologne : CLUEB.

MANDICH, Anna et PELLANDRA, Carla (dir.) (1991). Pour une histoire de l'enseignement du français en Italie. Actes du Colloque de Parme 14-16 juin 1990. Documents pour l'histoire du français langue étrangère ou seconde, 8. Paris : SIHFLES.

MINERVA, Nadia et PELLANDRA, Carla (1991). Insegnare il francese in Italia-Repertorio di manuali pubblicati dal 1625 al 1860. Bologne : Patron Editore.

MINERVA, Nadia (1991). Insegnare il francese in Italia - Repertorio di manuali pubblicati dal 1861 al 1922. Bologne : CLUEB.

VIGNER, Gérard (2016). «L'exercice ». Carnets Deuxième série - 8. Revue électronique d'Études françaises. A.P.E.F. En ligne : [http://journals.openedition.org/carnets/1850].

\section{ANNEXES}

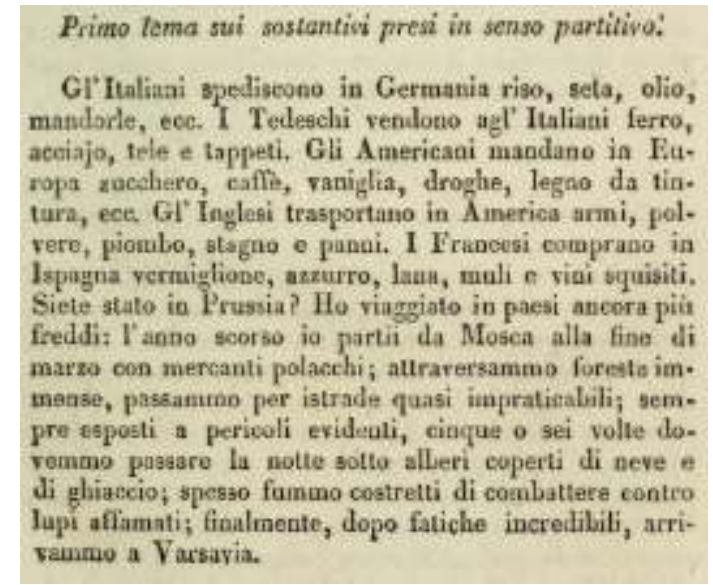

Document 1 : (Torretti $1823: 48$ ) 


\section{Esercizio 53}

\section{Casi particolari del partitivo}

Non bevo latte, ma birra. Con questo caldo, non si fa altro che bere. Alla televisione egli non guarda che documentari. Voi non avete sentimenti volgari. Il suo modo di vivere è un po' strano: vive senza amici, senza distrazioni, senza interessi. Voi non sapete tradurre due parole senza fare errori. Voi non avete né amici, né danaro, né protettori, come sperate di ottenere con facilità questo impiego? Egli corrisponde con numerosi amici d'ogni paese e lo fa sempre con piacere, con esattezza e con risultati notevoli. Come, non vi divertite? Non avete denaro?! Abbiamo mangiato riso con piselli. Voi avete molti debiti, troppi debiti, mio caro signore. Mario ha molti difetti, ma ha anche molte virtú. Voi, non avcte abbastanza coraggio per affrontare e superare queste difficoltà. Voi leggete pochi libri, inoltre ne leggete piú cattivi che buoni. Il grande e largo viale era imbandierato: ban- 24 a tre diere ad ogni finestra, fiori ad ogni balcone. Avete ragione, in queste pavvisé condizioni non vi resta che chiudere bottega.

Document 2 : (D’Alessandro 1973 : 69)

69 Traduire les phrases suivantes.

1. Aspettami, devo comprare le sigarette.

2. Gli impiegati della banca mettono quasi sempre la cravatta.

3. I dietologi dicono che dopo i cinque anni non si deve più bere il latte.
4. Non posso dormire: ci sono i vicini che fanno rumore.

5. Cosa prendi, la pasta o il riso?

6. Hanno dovuto leggere molti libri per scrivere quest'articolo.

\section{Même exercice.}

1. La signora Martin non vuole animali in casa.

2. Sbrigati, sono già le otto, siamo in ritardo.

3. Veniva a Fontamara quando gli uomini erano al lavoro e nelle case non trovava che donne e creature (SILONE).

4. Parlarono per ore, arrivando alla conclusione che [...] il dipinto era stato [...] un'attività marginale, quasi un capriccio (SCIASCIA).

5. Ho già detto che sugli alberi noi trascorrevamo ore e ore, e non per motivi utilitari come fanno tanti ragazzi, che ci salgono solo per cercare frutta o nidi d'uccelli (Calvino).

6. La mia isola ha varie spiaggie dalla sabbia chiara e delicata, e altre rive più piccole, coperte di ciottoli e conchiglie, nascoste tra grandi scogliere (MORANTE). 
3. VERSIONE ORALE O SCRITTA.

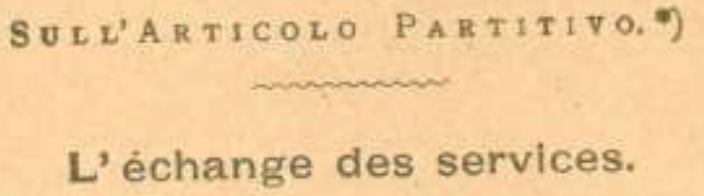

Voici un homme appartenant i) a une classe modeste de la sociéte, un menuisier de village, par exemple; observons tous les services qu' il rend i la sociteté et tous ceux qu' il en reçoit a); nous ne tarderons pas à être frappés de $\mathrm{I}^{2}$ énorme disproportion apparente

Cet homme passe sa journée a raboter des planches, a fabriquer des tables et des armoires; il se plaint 3 ) de sa condition et cepcúfant que reçoit-il de cette société en échange de son travail ?

D' abord 4), tous les jours, en se levant s), il s' habille, et il $\mathrm{n}^{\prime}$ a bit 6) aucune des nombreuses pièces 7) de son vétement. Or, pour que 8) ces vétements soient 9) ì sa disposition, il faut 10) qu' une

enorme quantité de travail ait été iI) accomplie. Il faut que des Amo ricains aient produit $\mathrm{a}^{2}$ ) du coton; des Indiens, de $l$ indigo; des Francais, de la laine et du lin; des Brésiliens 13), du cuir; que tous ces matériaux 14) aient été 15) transportés en des villes diverses; qu' ils aient été ouvrés, filès, tissés, teints ${ }^{16}$ ), ete. ${ }^{17}$ ). Pour que le pain ${ }^{8}$ ) qu' il mange lui arrive 19) tous les matins, il faut que les terres aient étb défrichées, fumies 20), labourèes, ensemencées, etc.

F. Bastlat *)

Document 4 : (Bassi 1912 : 8-9)

\section{ESERCIZIO SCRITTO.}

\section{Sull' Articolo Partitivo.}

Camericre 1), datemi э) prosciutto, burro, pane e birra, desidero far colazione 3 ): mi porterete 4) anche arrosto di vitello con insalata, poi pesche e formaggio. Le porteró 5) buon vino bianco, poichè non abbiamo 5) birra. Chi sono quei signori r)? Sono 0) negozianti francesi ; essi vengono 9) spessissimo :0) in questa u) citti, per comprare vino, seta, acciaio e droghe. Bisogna 13) amare le persone che ci danno $(x)$ buoni consigli ed eccellenti esempi. Cameriere 1), avete cartoline postali 44)? Non ho is) cartoline postali, ho soltanto fransobolli 16). - Proverblo: Gli amici son buoni in ogni piazka, o d ben aver degli amici per futto, ${ }^{7}$ ) (Giust).

1) Camariere, garpon. - 2) Datami, donnez-moi. - 3) Deridaro far cola. sione, je désire déjeuner. - 4) $M i$ porterste, vous m'apporterez. 5) Ls forturd, je vous apporterai, - 6) Poichd now abbiamo, oar nous ' 8) Sono, in quesio caso ; co sont. - 9) (Fengano, viennent. - 10) Spessissima, (1) gucsta, dans ootte. - 12) Bitogua, il faut. - 13) Che ci trés souvent. - (5) Now he, danne, qui nous donnent. - H t Crimbre-poste 3 francobolli, +imbres-posto. jo n' ai pas. - 16) Jranccobollo, timbos amis partout.

t7) Psovense: II est bon d'avoir des amis part

Document 5 : (Bassi 1912 : 9) 


\section{Sull'articolo partitivo.}

I. Dalla finestra io vedo (je vois) monti, valli, prati, boschi e case. Scorgo anche ( $j$ aperçois aussi) dei bei (beaux) giardini con dei bei (belles) fiori.

2. Il cartolaio vende (vend) carta, penne, inchiostro, matite ed altri oggetti.

3. Nella casa del ricco vi sono (il y a) numerose stanze con bellissimi (très beaux) mobili.

4. Voi cercate (cherchez) sempre dei pretesti.

5. Non cercate (ne cherchez pas) sempre pretesti. Non domando (demande) consigli; voglio (je veux) degli esempi, dei buoni esempi:

6. Non bevo ( $J e$ ne bois pas) vino; non bevo mai (je ne bois jamais) liquori.

7. Hai avuto brutti voti (mauvaises notes) alla scuola?

8. Avevate ragione; Luigi, invece (au contraire) aveva torto.

9. Abbiate (prenez) pazienza, non abbiate fretta (hâte).

Io. Le ricchezze sono buoni servitori (serviteurs), ma cattivi padroni (maitres).

II. Una casa senza libri (sans livres) è una povera casa.

I2. Egli ha fratelli e sorelle.

I3. Portate (apportez) sigarette anche per me (moi).

Document 6 : (Bosisio 1949b : 13)

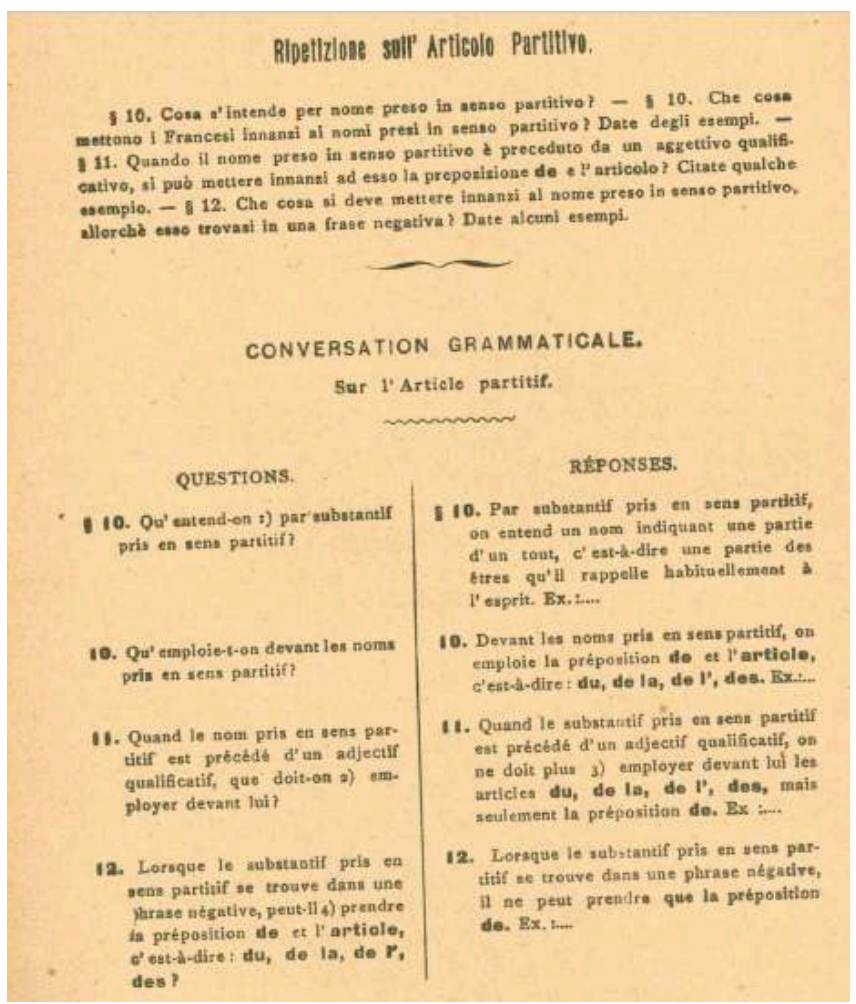

Document 7 : (Bassi 1912: 10) 
57 Compléter les phrases suivantes.

1. Est-ce que tu veux bière?

2. II reste encore caviar et champagne au buffet.

3. Ils ont apporté lertre aux lapins.

4. Il $\mathrm{y}$ avait bien soleil, mais la température était basse.

5. Il lui a fallu ............... patience pour finir son puzzle.

6. Nous avons eu ................. mal à les trouver, ils habitent un endroit isolé.

7. Sattends __............ courrier, c'est urgent.

8. Il a encore perdu _............. terrain sur ses poursuivants.

9. Elle nous jette __._._.... poudre aux yeux, mais cela ne nous impressionne pas.

Document 8 : (Bidaud 1994b : 24)

8 Completa con le forme appropriate ( $d u$, de la, औth de $l^{\prime}$, des, de, $\left.d^{\prime}\right)$ quando è necessario.

1 Prends ............. argent dans mon porte-monnaie et va acheter un peu .............. pain, s'il te plaît.

2 Vous avez eu vraiment beaucoup chance.

3 Écoutez avec ….......... attention, sans prendre notes.

4 Mathilde boit toujours un bol .............. lait le soir.

5 À mon avis, il y a peu ............... crêpes. Je vais préparer ...............autres crêpes.

6 Vous prenez encore un peu ………... poisson?

7 Elle est végétarienne et elle ne mange ni viande ni …........ jambon.

8 Il faut conduire avec beaucoup .............. prudence.

9 Tu vas être malade! Tu manges trop ……....... sucreries.

10 Vous avez ........ raison, mais il faut parler avec prudence.

11 Vous nêtes plus .............. enfants ! Jouez sans faire bruit!

12 Je prépare mon gâteau avec crème Chantilly.

Document 9 : (Beneventi $2011: 87$ ) 


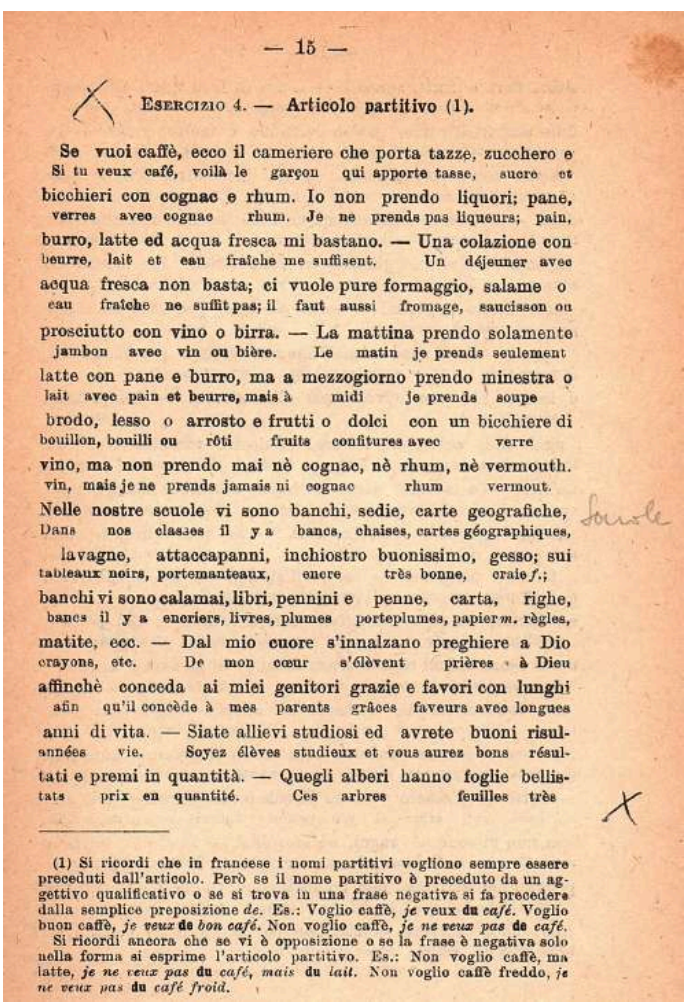

Document 10 : (Truchi $1949: 15)$

58 Compléter et indiquer s'il s'agit de l'article contracté ou du partitif.

1. II souffre estomac.

2. Elle se fait _._...... souci pour son fils qui n'est pas encore revenu.

3. On joue __...... Pirandello sur scène en ce moment.

4. Il joue ........... harpe.

5. Je lui ai arraché la feuille

6. Ils ont arraché _..._._..... plants de vigne.

7. Nous avons découpé __.......... viande pour le dîner.

8. Elle s’est éloignée ..._......... amis qui Tavaient aidée.

9. À Noël, on accroche _...__._. houx sur le sapin.

10. Les rideaux se sont décrochés __........... fenêtre.

11. Il ne parvient pas à détacher son regard _........... rivage.

12. Nous attachons parfois __.......... importance à des objets qui n'en valent pas la
peine.

Document 11 : (Bidaud 1994b : 24)

\section{NOTES}

1. N. Minerva et C. Pellandra ont entrepris il y a une trentaine d'années une recherche de grande ampleur consistant à répertorier tous les ouvrages de français à visée pédagogique édités en Italie depuis le début de l'enseignement de la langue française dans le pays. Elles ont ainsi conçu deux répertoires : le premier couvre les années 1625 à 1860 ; le deuxième, les années 1861 à 1923 (uniquement sous la direction de Minerva) et Anna Mandich a dirigé un troisième ouvrage couvrant la période de 1923 à 1943. 
2. Terme entendu dans le sens de « description d'une régularité de la langue » (Cuq 2003 : 213).

3. Les titres ont été maintenus dans les langues utilisées par l'auteur.

4. H. Besse et R. Porquier emploient les mots « tâche » et « activité » comme synonymes.

5. Les textes s'apparentent à des leçons de morale. À 30 ans d'intervalle, des textes identiques sont présents chez les deux auteurs.

6. Le premier manuel, rédigé par Pietro Durante, a été imprimé en 1625 sous le titre La grammatica italiana per imparare la lingua francese.

7. D’après R. Lado (1971 [1957]), l'analyse contrastive doit mettre en évidence les différences et les ressemblances entre deux langues décrites structuralement, point par point, les écarts entre les deux langues constituant les points de difficulté pour les apprenants.

8. Les traductions sont de l'auteure.

9. Dans la troisième édition datant de 1829 , il en propose trois.

\section{RÉSUMÉS}

L'article a pour objectif de dégager certaines caractéristiques des exercices de français conçus pour les élèves italiens depuis leur généralisation dans les manuels de langue au XIX ${ }^{\mathrm{e}}$ siècle. L'auteure donne une dimension historique à sa recherche menée à titre exploratoire en s'appuyant sur un corpus composé d'exercices autour de l'article partitif, tirés de sept ouvrages d'enseignement/apprentissage du français édités entre 1823 et 2011. La mise en perspective de la notion d'exercice avec le corpus, l'identification des types d'exercice présents à partir de la typologie de Gérard Vigner (2016) et l'analyse qualitative des exercices conduisent à deux constats. D'une part, les exercices présentent deux caractéristiques constantes : le recours à la traduction et l'approche contrastive. D'autre part, ils suivent l'évolution de l'enseignement/ apprentissage des langues dans leur diversification en termes de tâches à effectuer par l'élève.

The aim of the article is to identify some characteristics of exercises designed for Italian pupils since their generalization in handbook of French in the nineteenth century. The author gives a historical dimension to her exploratory research based on a corpus of exercises around the partitive article, from seven French teaching/learning handbooks published between 1823 and 2011. Putting into perspective the concept of exercise with the corpus, identifying types of exercise based on the typology of Gérard Vigner (2016) and analyzing the quality of the exercises lead to two observations. In the first place, the exercises have two constant characteristics : the use of translation and the contrastive approach. Secondly, they follow the evolution of language teaching/learning in their diversification in terms of tasks to be performed by the pupils.

\section{INDEX}

Mots-clés : article partitif, approche contrastive, types d'exercice, français pour italophones, traduction

Keywords : partitive article, contrastive approach, types of exercise, French language for Italian speakers, translation 
AUTEUR

RAPHAËLE FOUILLET

Centre de Recherche en Éducation de Nantes

raphaele.fouillet@univ-lemans.fr 